\title{
Du travail filmé au travail du film
}

\section{Baptiste Buob et Jean-Paul Géhin}

\section{OpenEdition}

Journals

Édition électronique

URL : http://journals.openedition.org/itti/277

DOI : $10.4000 /$ itti. 277

\section{Éditeur}

Université de Poitiers

\section{Référence électronique}

Baptiste Buob et Jean-Paul Géhin, « Du travail filmé au travail du film », Images du travail, travail des images [En ligne], 8| 2020, mis en ligne le 01 février 2020, consulté le 14 avril 2021. URL : http:// journals.openedition.org/itti/277 ; DOI : https://doi.org/10.4000/itti.277

Ce document a été généré automatiquement le 14 avril 2021.

Images du travail, travail des images 


\title{
Du travail filmé au travail du film
}

\author{
Baptiste Buob et Jean-Paul Géhin
}

1 Un des contributeurs de ce numéro propose de définir simplement le travail comme une activité qui nécessite l'engagement de certaines capacités physiques et cognitives afin de créer des choses et/ou des idées nouvelles. Cette définition, au demeurant très banale, a l'avantage de pouvoir être appliquée de façon symétrique aussi bien à ce qui peut intéresser un chercheur (le travail comme objet de recherche) qu'à sa propre activité (le processus de recherche comme travail). C'est une comparable acception qui a guidé l'élaboration de ce numéro où les contributeurs n'ont pas toujours le travail comme objet central de recherche mais où tous exposent de façon réflexive la façon dont ils travaillent avec la caméra et le film.

Dans le sillage du troisième numéro de la revue qui s'intéressait aux usages et aux statuts des images dans le processus d'enquête, il s'agit donc ici non seulement de considérer différentes façons de faire de la recherche filmée sur le travail, mais aussi de mettre l'accent sur « les effets de la fabrication ou de l'usage de ces "données visuelles" sur les situations d'enquête" (Meyer \& Papinot 2016: \$5) en mettant la focale sur l'utilisation de caméras et d'images animées. Ce numéro réunit cependant des textes plus contrastés : diverses approches disciplinaires, différentes acceptions de la notion de «travail» et autant de fonctions dévolues à l'image. Se côtoient ainsi des contributions émanant de diverses disciplines - anthropologie, sociologie et géographie (ainsi qu'un varia en psychologie du travail) - rédigées soit par des « novices » - s'étant engagés pour la première fois dans la réalisation d'un film - soit par des chercheurs plus confirmés - ayant élaboré au long des années une réflexion méthodologique faite de procédures et de cadres théoriques éprouvés (fussent-ils très souples).

3 Par la mise en regard de ces textes - mais aussi à la lecture du compte-rendu du film de Pascal Césaro (Une maison au bord du monde, 74mn, Production du Lagon, 2018) et du grand entretien avec Bernard Ganne qui accompagne le dossier thématique -, trois traits se dégagent plus particulièrement. Il ressort tout d'abord que chaque contributeur aborde le travail selon des perspectives spécifiques et déploie des méthodes filmiques singulières pour appréhender un aspect particulier du complexe phénomène travail. On constate que si le film a fait ses preuves pour étudier le travail 
selon certaines perspectives canoniques, de nombreux chercheurs y recourent afin de replacer les activités de travail dans un environnement sensible et écologique plus large. Force est ensuite de remarquer que tous les textes font état d'approches qui se démarquent de certaines pratiques où la caméra est envisagée comme outil rigoureux pour étudier le travail. Plaçant la relation qu'ils nouent sur le terrain au centre de leurs préoccupations, tous ont tourné le dos a une approche naturaliste et font de la réflexivité un aiguillon de leur recherche. Enfin la notion de partage apparaît comme étant un dénominateur qui tend à devenir commun : le singulier partage de l'espace que permet l'expérience de tournage s'accompagne de plus en plus de pratiques qui relèvent des sciences participatives où il s'agit de faire du processus de recherche un commun partagé avec les personnes filmées. Cependant, nous le verrons, ces approches présentées comme innovantes ont des précédents désormais presque centenaires. Aussi les efforts sont encore à poursuivre pour qu'un réel cumul des savoirs concernant l'utilisation du film puisse se faire entre les disciplines et donc que le partage se fasse entre ces chercheurs qui s'apparentent eux-mêmes, par certains aspects, à des travailleurs.

\section{Tel film, tel travail}

Georges Friedmann estime que l'étude du travail ne peut être le propre d'un seul domaine disciplinaire des sciences humaines et sociales - le "phénomène global» devrait selon lui être considéré à partir de différents points de vue complémentaires et définit cinq principaux angles d'attaque possibles pour le considérer: technique, physiologique, psychologique, social et économique (Friedmann 1960). Naturellement, une telle partition propose un modèle abstrait car il serait artificiel de vouloir séparer ces domaines forcément intriqués. Cependant cette idée suivant laquelle le travail peut être abordé selon diverses perspectives nous intéresse d'autant plus ici que Georges Friedmann considère que cet exercice revient à " examiner le travail comme avec une caméra, sous des angles successifs et différents » (ibid. : 690). Cette comparaison n'est pas de simple rhétorique : le langage cinématographique procède généralement ainsi, par un jeu d'inclusion/exclusion, et tend à souligner certaines manifestations tangibles des situations au détriment d'autres qui seront, de fait, masquées ou estompées suivant des lois relevant de la scénographie générale du cinéma (X. de France 1982). Par exemple, de façon schématique, saisir en gros plan l'action d'une scie sur une branche interdit de voir l'arbre auquel elle appartient, le(s) corps qui tien(nen)t l'outil et l'environnement écologique général dans lequel ils s'inscrivent. Ainsi le chercheurcinéaste aura tendance à mettre l'accent sur une ou quelques dimensions du travail, à affirmer donc un point de vue sans prétendre saisir le phénomène dans sa globalité (et ce d'autant plus facilement que certaines perspectives de l'étude du travail ne relèvent pas du domaine du visible et de l'audible privilégiés par l'appréhension filmique). La plupart des contributeurs du numéro proposent ainsi de considérer le travail selon des angles singuliers, insistant chacun sur la capacité du film à mettre l'accent sur un de ses aspects saillants.

5 Pour Baptiste Buob, formé à l'anthropologie des techniques, le film est notamment utilisé afin de saisir des actes de transformation dans leur dimension processuelle. En décidant, lors de ses premiers travaux, de faire de l'objet en cours de transformation le fil conducteur de ses films, il parvient à saisir la technique à l'œuvre, suivant le constat 
fait par André Leroi-Gourhan de la capacité unique du film à décrire le «drame » du « jeu de l'homme et la matière » (Leroi-Gourhan 1983 [1948] : 63). Cependant il ne s'agit plus ici d'écrire un script a priori comme le proposait ce dernier, mais de s'engager, en temps réel, dans des formes d'improvisation filmiques. La subjectivité inhérente au choix effectués par l'opérateur est considérée comme une ressource utile pour appréhender la situation filmée, participant même d'une transformation heuristique. Le film est alors assumé comme étant le fruit d'une élaboration plurielle où non seulement filmant et filmés, mais aussi le futur spectateur virtuel, participent de la création d'objets singuliers qui permettent de rendre compte du monde sous des angles inattendus et de révéler certaines dimensions culturelles et sociales autrement inaccessibles. Annalisa Lendaro, bien qu'elle se soit davantage intéressée au travail du film qu'au travail filmé, a la même intuition lorsqu'elle remarque la présence d'un « changement de ton ou même de contenu dans la parole enregistrée devant la caméra par rapport à celle livrée hors enregistrement ». Constat fait de ces modifications, elle a alors invité ses interlocuteurs à échanger avec elle sur « ce qui se dit et ce qui ne se dit pas en public », soulignant également l'heuristique d'un usage conjoint de l'outil filmique et de l'entretien classique.

6 Cette préoccupation pour le régime d'engagement des personnes filmées fait directement écho à la contribution de Christian Lallier, davantage influencé par une conception interactionniste $\mathrm{du}$ travail que par l'anthropologie des techniques. Préoccupé par « l'état de réflexivité produit par l'engagement d'une personne dans son action » et reprenant la distinction canonique établie notamment par l'ergonomie entre travail prescrit et travail réel, Christian Lallier s'intéresse à "ce qui se joue » entre les acteurs dans une situation. Il ne s'agit pas tant pour lui d'enregistrer les gestuelles et les pratiques professionnelles que de saisir les relations sociales induites par les situations de travail en rendant compte de « ce que (se) disent les acteurs entre eux ». Selon cette perspective, l'acte filmique a pour principal mission de rendre compte de la parole des acteurs, celle-ci étant la condition nécessaire pour « donner à comprendre les enjeux qui motivent leurs engagements dans la situation sociale observée ».

7 Ces deux angles d'attaques, sous-tendus par des relations contrastées des chercheurs avec les personnes filmées, offrent des portes d'entrée bien différentes sur les situations de travail. Cependant il ne s'agit pas ici uniquement de choix et de regards reposant sur des préoccupations épistémologiques différentes. En effet, si chacun prend un parti théorique, encore faut-il qu'il puisse filmer des situations qui se prêtent à ses hypothèses de travail. Si pour Baptiste Buob c'est principalement l'action en train de se faire qui primera pour considérer l'activité technique, pour Christian Lallier ce sont davantage les phases qui précèdent et qui suivent les actions techniques qui seront privilégiées. Mais en définitive il s'agit bien d'appréhender le même objet, l'un mettant le curseur sur le social, l'autre sur le technique, tout en sachant que l'intrication de ces perspectives est seule à même de rendre compte du phénomène puisqu'il n'est pas possible de saisir le technique sans saisir le social, pas plus qu'il n'est possible de saisir le social sans le technique. Il y a donc bien certaines dominantes des activités selon les moments et les situations, des aspects qui s' « auto-soulignent», - des aspects qui "se mettent en valeur d'eux-mêmes de façon ostensible et souvent prévisible » - et dont le cinéaste aura tendance à rendre compte de façon privilégiée (Cl. de France 1989 [1982] : 368). 
8 Hannelore Girardot-Pennors et Marie Chenet s'intéressent également, bien que de façon moins directe, à des situations de travail. Par leurs approches, elles soulignent principalement que les actions humaines s'inscrivent dans des écosystèmes complexes, entretenant des relations continues avec l'environnement. En filmant l'activité quotidienne dans une petite épicerie, la première en vient à faire le portrait d'un espace en relation continue avec une ville et l'histoire de ses habitants. Depuis ce lieu, où se déploient des activités du secteur dit «tertiaire ", la cinéaste a choisi de souligner la richesse des relations humaines qui unissent les individus partageant un même territoire, les gestes du travail devenant alors un prétexte.

9 Marie Chenet est également attentive à l'écologie humaine qui accompagne certaines situations de travail soulignant comment l'environnement l'affecte et que le film est un outil de recherche approprié afin d'en rendre compte. La géographe recourt ainsi à l'usage de la caméra pour éprouver certaines préoccupations actuelles de sa discipline "afin de produire et de formaliser de façon singulière des savoirs géographiques ", nous dit-elle -, telles la relation des individus à l'environnement, l'« expérience spatiale des corps » et les émotions. C'est ainsi, par exemple, que l'on prend conscience que le quotidien d'un berger est peuplé d'une multitude d'entités dont certaines, bien qu'essentielles, ne sont pourtant jamais visibles, tels le loup ou des catastrophes naturelles, peuvent avoir sur un territoire et certaines de ses activités économiques.

Ayant fait ses preuves dans les domaines de l'appréhension des dimensions techniques et sociales du travail, le film est désormais employé pour appréhender des relations moins directement perceptibles. La mise en regard de ces diverses contributions montre ainsi que si le film permet d'appréhender le travail depuis des perspectives canoniques et obvies, il est désormais couramment utilisé pour saisir des aspects plus sensibles des activités humaines en les inscrivant dans des espaces plus larges et des relations plus fines.

\section{Filmer en cherchant}

Abordant le travail selon des perspectives contrastées, les approches réunies dans ce numéro ont en commun d'affirmer avoir tourné le dos à une approche strictement spéculaire du film et donc à une forme de rationalité qui se retrouve dans les premiers usages de l'image à fin scientifique, notamment parce que chacun conçoit son influence sur les situations et s'affirme comme "auteur " prétendant parfois réaliser des films « de création ». L'articulation étroite et l'enrichissement réciproque entre recherche et réalisation semble ici être désormais un acquis. On mesure alors le chemin parcouru depuis deux décennies lorsqu'un numéro de la revue Champs visuels traitant du même thème séparait la réflexion en deux parties (images brutes du travail dans la première partie rédigée principalement par des chercheurs; travail de l'image dans laquelle interviennent surtout des réalisateurs) et quand la revue Langage \& Travail réduisait l'image à un outil d'" amplification des conditions analytiques ", comme si le film était à l'activité, ce que le magnétophone est à la parole (Lacoste, 1996 : 8).

Cependant s'il est aujourd'hui parfois de bon ton en sciences humaines de se démarquer de telles approches qualifiées péjorativement de positivistes, il serait totalement idiot de les rejeter en bloc tant elles apparaissent utiles à l'étude du phénomène travail. Le varia écrit par Marc Durand, Annie Goudeaux, Germain Poizat et Juana Sarmiento Jaramillo expose par exemple une utilisation pointue et spécifique de 
la réalisation filmique dans le cadre d'une enquête menée avec des huissiers à des fins de formation professionnelle. S'inscrivant dans la longue tradition d'utilisation du film d'élicitation en psychologie (Gross et Levenson, 1995), les auteurs recourent aux images animées pour perturber les agents, les incitant à une forme d'« engagement mimétique " couplée à une forme spécifique de "reenactement», afin qu'ils se réapproprient leurs activités professionnelles. Ainsi trouve-t-on ici (à l'instar d'autres démarches appliquées en psychologie, en ergonomie ou en sciences de l'éducation par exemple) des usages de la caméra qui visent non seulement à mieux comprendre le travail - par une implication expérimentale des agents -, mais aussi à accompagner un changement de perception de leurs activités. Les auteurs réunis dans le dossier principal utilisent quant à eux la caméra tout au long de leurs recherches et conçoivent leur approche comme étant davantage cinématograhique.

Certes les contributeurs s'affirment cinéastes, mais ils utilisent également leurs images de façon rétrospective pour étudier les situations filmées. Car l'image conserve toujours une valeur proprement documentaire, elle garde trace de quelque chose, le fameux « ça a été » de Roland Barthes. Luc de Heusch relate que l'«éternel dilemme entre le document pur et son élaboration, entre la science et l'art» préoccupe déjà les chercheurs dans les années 1950 : pour certains les images ne devaient constituer qu'un "complément du carnet de notes" tandis que d'autres "voulaient aboutir à une écriture plus recherchée » (de Heusch 2006 : 46). Mais selon lui cette tension n'est pas vraiment pertinente, rappelant que même l'ethnographe utilisant un carnet ne fait pas autre chose qu'élaborer « sous forme d'article ou de livre, les données brutes recueillies sur le terrain » (ibid.). Pour les contributeurs ici réunis, il s'agit également de chercher à associer les deux dimensions. Même s'ils visent à "faire des films", ceux-ci se construisent bien à partir d'images qui constituent autant de "notes de terrain». Autrement dit, si par ces réalisations leurs auteurs souhaitent qu'elles soient diffusées au-delà des cercles spécialisés, elles procèdent cependant d'une démarche pleinement exploratoire et la découverte des situations filmées se fait en « temps réel ».

La différence avec des approches objectivistes repose notamment sur le fait que le chercheur, qui est le plus souvent son propre opérateur, revendique sa contribution à la construction d'un point de vue: "L'ethnographe-cinéaste affirme ainsi sa subjectivité et son rôle dans la transformation du monde par sa mise en image » nous rappelle Baptiste Buob en soulignant que le tournage ne se limite pas à capter des situations mais permet de proposer des interprétations et devient alors un «acte singulier de mise en forme ». Conscient de son rôle dans le processus de constitution des données, le cinéaste prend parfois le parti de s'inscrire dans l'objet de son étude afin de mettre au jour les soubassements du travail d'enquête comme il est désormais d'usage dans les études faisant de la réflexivité un embrayeur central de leur approche (Ghasarian 2002). Ainsi Hannelore Girardot-Pennors vise-t-elle à rendre sa présence et son statut explicites pour le spectateur. Marie Chenet affirme sa subjectivité lorsqu'elle parle à la première personne, comme toute fraiche diplômée et jeune encadrante dans Apprentis chercheurs, documentaire présentant la formation sur le terrain des étudiants en géographie; suivant une tendance désormais ancrée dans l'histoire du film ethnographique, « la relation instaurée dans le cadre d'une situation anthropologique » devient alors le sujet principal du film (Piault 2000 : 70).

15 Jay Ruby rappelle que la réflexivité s'est progressivement imposée comme garde-fou éthique et méthodologique et s'est accompagnée du désir de cinéastes de révéler à 
l'audience la dimension construite et les hypothèses épistémologiques sous-jacentes des réalisations audiovisuelles (Ruby $2000: 156$ ). La réflexivité ne se limite plus dès lors à la nécessité de révéler les soubassements de l'enquête et leurs influences sur les connaissances produites : la réflexivité se trouve intégrée dans le film lui-même afin d'expliciter l'approche du chercheur et révéler le processus dialogique de construction du savoir (Pink 2003 : 189). Comme le note Marie Chenet, il s'agit de répondre aux exigences de transparence et de réflexivité de disciplines qui «privilégient un regard sur une réalité observée, s'interrogent sur la construction de cette réalité et explicitent les conditions précises selon lesquelles le chercheur se livre à cet exercice ». Ces façons de rendre compte de la subjectivité des cinéastes comme des enquêtés sont ainsi des manières d'affirmer que la démarche documentaire procède d'une coproduction, rejetant l'idée d'une possible capture d'une réalité existant indépendamment des relations qui se tissent lors de son élaboration.

16 Pour qui est rompu à la pratique audiovisuelle, ces considérations ne sont pas particulièrement originales. Cependant, quitte à céder à un certain psittacisme, il paraît encore nécessaire de les répéter quand on constate, avec Baptiste Buob, qu'au sein même de la discipline anthropologique, que l'on pourrait croire vaccinée contre ce genre de procès, l'utilisation d'une caméra reste parfois associée par des chercheurs up to date à une approche naturaliste.

\section{Le film en partage}

17 Les contributeurs au dossier constitué pour ce numéro semblent avoir fait leur, l'appel au décloisonnement du film scientifique par son rapprochement avec le documentaire de création que Jean-Paul Colleyn appelait notamment de ses vœux il y a près de trente ans (Colleyn 1992 : 7). Pour autant si l'on ne peut que souscrire à cette orientation qui rappelle la conception du film ethnographique promue par Jean Rouch - lequel souhaitait que soit associée «la rigueur de l'enquête scientifique à l'art de l'exposé cinématographique» (Rouch, 1968: 463) comme le rappelle Hannelore GirardotPennors -, force est de constater que si la plupart des contributeurs ici réunis ne réduisent pas les images à « des matériaux fiables » (Lacoste, 1996 : 11), ils ne réalisent pas pour autant des films qui s'inscrivent pleinement dans les circuits de production et de diffusion du cinéma documentaire. Comme le propose également Hannelore Girardot-Pennors, ces réalisations relèvent davantage de la catégorie des films dits de «néo-exposition»: chaque réalisation associe exploration et langage cinématographique et constitue ainsi «un document méthodologique irremplaçable sur l'aventure d'un procès d'observation» ( $\mathrm{Cl}$. de France 1989 [1982]: 348). La réalisation du film n'est pas toujours ici une fin en soi, c'est aussi - voire avant tout un outil méthodologique de l'enquête qualitative et le produit d'une recherche. C'est alors davantage la question de savoir ce que le film fait à la recherche, la façon dont le film travaille la recherche, qui est au centre des contributions.

La caméra, on le sait, est un précieux outil de négociation pour entrer sur le terrain et construire la relation d'enquête, qu'il s'agisse d'occuper le statut d'un "petit " qui conforte les personnes filmées dans leurs positions, comme le conçoit Christian Lallier, ou de s'engager dans des formes de présence plus affirmatives, faisant du cinéaste un acteur à part entière, agissant sur les situations. Ainsi, contrairement à l'idée encore répandue que la caméra constitue une gêne dans la démarche d'enquête, tous les 
articles de ce dossier soulignent qu'elle conduit à penser et à négocier sa place sur le terrain. Le film, comme toute méthode d'enquête plus ou moins participante sans caméra, repose sur le partage d'un espace et induit différents types de coprésence. Cependant le film a cette particularité, lorsque le cinéaste s'implique corporellement dans les situations, d'induire des formes particulières d'engagement dont on découvre ici plusieurs exemples, allant de présences silencieuses (Lallier, Buob) aux échanges dialogiques (Chenet, Girardot-Pennors, Lendaro). Suivant des constats faits à maintes reprises, le recours à une caméra permet aux chercheurs d'occuper des espaces auxquels ils n'auraient pas pu accéder autrement.

Il ne s'agit alors pas tant ici de se faire oublier que de trouver une place qui fasse sens pour les personnes filmées lesquelles intègrent d'une façon ou d'une autre le cinéaste dans leur système de représentation malgré le fait qu'il exerce une pratique technique indépendante qui lui interdit de participer aux mêmes activités qu'eux. En conséquence chaque nouvelle expérience de tournage nécessite de faire des choix et d'élaborer des méthodes singulières. C'est ainsi que Marie Chenet et Baptiste Buob font état de formes d'engagement très différentes selon les contextes et les objectifs. On découvre alors ce dernier changer radicalement sa pratique en adoptant le tournage en plans-séquences afin de se mettre au diapason d'un processus de création en danse contemporaine. Pour jouer sur les mots, le cinéaste se doit d'entrer dans la danse, laquelle lui dicte, au moins pour partie, la marche à suivre.

Dans les contributions ici réunies, les formes de participation vont bien au-delà de la simple coprésence lors de l'acte filmique: l'autorité est partagée entre filmants et filmés. On constate en effet qu'un ensemble de pratiques dialogiques prenant appui sur le film tendent à se généraliser en sciences humaines : le film est reconnu comme un outil particulièrement heuristique dans le cadre de ce que l'on qualifie aujourd'hui comme relevant des méthodes participatives. Au côté du recours désormais classique à l'utilisation du film comme "guide d'entretien" afin de questionner les personnes filmées - dont on date l'émergence du milieu des années 1960 en ethnographie (Cl. de France, 1989 [1982] : 305) et qui suit des protocoles très stricts dans d'autres approches comme en témoigne l'article de Durand et al. publié en varia -, on observe de plus en plus de chercheurs projeter leurs images afin de questionner leur démarche elle-même. C'est ainsi que pour Hannelore Girardot-Pennors, l'introduction de la caméra implique d'exposer sa démarche au triple sens du terme à savoir: la montrer, l'expliciter et la mettre en danger. Il s'agit donc d'impliquer les personnes filmées dans le processus de recherche comme le fait également, d'une autre manière, Annalisa Lendaro en mettant son film au travail une fois terminé. Il s'agit alors de restituer aux principaux intéressés les résultats de la recherche mais surtout de la continuer et l'approfondir, le débat après la projection permettant d'affiner la compréhension des phénomènes étudiés et de dégager des nouvelles pistes de recherches. Ces diverses pratiques sont loin d'être nouvelles. Luc de Heusch soulignait déjà au début des années 1960 que Robert Flaherty avait développé depuis près de trente ans une méthode reposant sur " la collaboration effective des hommes dont le cinéaste veut exprimer les passions, les travaux et les soucis », ce qu'il qualifiait alors de " caméra participante » (de Heusch, $1962: 36-40$ ). Ce constat a de quoi surprendre: Luc de Heusch expose clairement les enjeux d'une méthode qui se développe dès les années 1930 et que certains voient aujourd'hui comme tout à fait innovante; ce qui, ceci étant, ne retire en rien de son intérêt. 
21 Nous voilà de nouveau confrontés à «l'étrange impression de revenir sans cesse au point de départ " dès lors qu'il s'agit de traiter de l'utilisation du film en sciences humaines (Cl. de France 1989 [1982] : XI). Ce constat tient selon nous à deux raisons principales : la première est que dans les disciplines instituées la réflexion sur l'usage du film est rarement transmise. Le chercheur qui se met subitement à utiliser une caméra peine à trouver des ressources dans son milieu et découvre par lui-même des effets épistémologiques qui viennent rompre avec certaines de ses certitudes. La seconde tient au fait que certaines disciplines sont en avance sur ces réflexions. Alors que l'anthropologie est de longue date au prise avec les questions d'audiovisuel, d'autres, comme la sociologie et la géographie, ne s'y intéressent sérieusement que depuis peu. Cet état de fait est surprenant quand on sait que des représentants de toutes ces disciplines ont réalisé des films dans le contexte bouillonnant du musée de l'Homme et qu'André Leroi-Gourhan posait alors les bases d'une réflexion pluridisciplinaire sur les usages du film en sciences humaines et sociales, dans un article d'ailleurs paru dans une revue d'ethnologie et de géographie humaine (LeroiGourhan 1983 [1948]). Le fait de voir se multiplier aujourd'hui de nouveaux espaces d'expression pluridisciplinaires (la présente revue, mais également la plus récente Revue française des méthodes visuelles par exemple) laissent espérer que ce nécessaire partage scientifique puisse être restauré au profit des réflexions sur l'utilisation du film.

22 Ce partage nous semble d'autant plus envisageable qu'il nous est apparu à lire ces différents textes que leurs auteurs s'apparentent peut-être plus à des artisanstravailleurs qu'à des artistes-esthètes. Comme le montre la sociologie des sciences depuis des décennies, le chercheur n'est pas hors du monde : engagé dans des réseaux de relations, ayant incorporé et transmettant des savoirs, il utilise divers outils et, comme tout un chacun, produit des choses nouvelles, affecte son environnement et est affecté par lui. Peut-être que l'appréhension du chercheur en sciences humaines et sociales comme travailleur à part entière est contrariée non seulement par certaines définitions étroites de la notion de travail mais aussi par l'apparente banalité et rareté des outils qu'il mobilise (carnet, stylo, ordinateur, etc.). Elle devient plus évidente lorsque celui-ci crée des films en utilisant une caméra et des logiciels de montage à l'instar de tous les contributeurs ici réunis.

En tant qu'homo faber, le chercheur-cinéaste est engagé dans une forme de travail, il exerce diverses actions qui agissent sur les situations filmées lesquelles, à leur tour, le modifie. Nous avons d'ailleurs ressenti à lire ces diverses contributions une certaine jubilation à filmer et à faire un film. Nous formulons l'hypothèse que ce plaisir provient justement de cet engagement corporel particulier du chercheur qui le contraint à observer à hauteur d'homme, et donc de rompre avec toute vision surplombante, tout en le conduisant à créer quelque chose en engageant tous ses sens. La recherche menée caméra au poing est une pratique où le plaisir, non réductible à l'obtention d'un objet fini, accompagne l'exercice de l'activité elle-même. Peut-être que le chercheur renoue ici avec la fascination qui accompagne les gens du faire lesquels, comme le dit John Dewey, ne peuvent rester indifférents au spectacle qu'ils contribuent à créer de leurs propres mains (2010 [1934] : 32). 


\section{BIBLIOGRAPHIE}

Colleyn J.-P. (1992), « Il faut décloisonner le genre ! », CinémAction, nº 64, p. 26-39.

Dewey J. (2010 [1934]), L'art comme expérience, Paris, Gallimard.

de France Cl. (1989 [1982]), Cinéma et anthropologie, Paris, Editions de la Maison des Sciences de l'Homme.

de France X. (1989), Éléments de scénographie du cinéma, Nanterre, Publidix.

Friedmann G. (1960), « Qu'est-ce que le travail ? », Annales. Economies, sociétés, civilisations, $\mathrm{n}^{\circ} 15$ (4), p. 684-701.

Ghasarian Ch. (2002), «Sur les chemins de l'ethnographie réflexive », in De l'ethnographie à l'anthropologie réflexive. Nouveaux terrains, nouvelles pratiques, nouveaux enjeux, Ghasarian C. (ed.), Paris, Armand Colin, p. 5-33.

Gross J. J. et Levenson R. W. (1995), « Emotion Elicitation Using Films », Cognition and Emotion, n 9 (1), p. 87-108.

de Heusch L. (2006), « Jean Rouch et la naissance de l'anthropologie visuelle », L'Homme, ${ }^{\circ}$ 180, p. 43-71.

de Heusch L. (1960), Cinéma et sciences sociales. Panorama du film ethnographique et sociologique, Paris, Unesco (« Rapports et documents de sciences sociales », $n^{\circ} 16$ ).

Lacoste M. (1996), «Filmer le travail pour l'analyser », Langage \& Travail, nº 8, p. 7-12.

Leroi-Gourhan A. (1983 [1948]), « Cinéma et sciences humaines. Le film ethnologique existe-til ? ", in Le Fil du temps. Ethnologie et préhistoire, Leroi-Gourhan A. (ed.), Paris, Fayard, p. 59-67.

Meyer M. et Papinot Ch. (2016), « Le travail des images dans la démarche de recherche. Analyse réflexive et compréhension de l'objet », Images du travail, travail des images, $\mathrm{n}^{\circ} 3$.

Piault M.-H. (2000), Anthropologie et Cinéma, Paris, Nathan.

Pink S. (2003). « Interdisciplinary agendas in visual research : re-situating visual anthropology », Visual Studies, $\mathrm{n}^{\circ} 18$ (2), p. 179-192.

Rouch J. (1968), « Le film ethnographique », in Ethnologie générale, Poirier J. (ed), Paris, Gallimard, p. $429-471$.

Ruby J. (2000), Picturing Culture: Explorations of Film and Anthropology, Chicago, University of Chicago Press.

\section{AUTEURS}

\section{BAPTISTE BUOB}

Formé à la fois à l'anthropologie et au cinéma, il est chargé de recherche au Laboratoire d'ethnologie et de sociologie comparative (CNRS/Université Paris Nanterre). S'intéressant aux articulations entre pratiques audiovisuelles et ethnographiques, ses recherches relèvent plus généralement de l'anthropologie des techniques et de l'anthropologie filmique. 


\section{JEAN-PAUL GÉHIN}

Jean-Paul Géhin est enseignant chercheur à l'Université de Poitiers et membre du GRESCO. Il a publié de nombreux articles et ouvrages en sociologie du travail et plus particulièrement sur la question des modalités d'articulation entre éducation et travail. Responsable et enseignant dans des masters de réalisation documentaire, il s'intéresse à la médiation scientifique et à l'usage des images en sciences sociales. Dans ce cadre, il a participé à la fondation en 2009 du festival Filmer le travail qu'il préside actuellement. Il fait également partie de la direction collégiale de la nouvelle revue numérique et pluridisciplinaire Images du travail, travail des images. 